\section{PTH-039 ENDOSCOPIC MUCOSAL RESECTIONOF DUODENAL ADENOMAS: SUCCESS, COMPLICATIONS, RECURRENCE, SURGERY-FREE OUTCOMES IN A UK TERTIARY-CENTRE}

Richard Lord*, Pradeep Mundre, Matthew Gold, Lawrence Hamshaw, Jack Kane, Noor Mohammed, Bjorn Rembacken, Ruch Sood. Gastroenterology department, Leeds NHS teaching hopsitals, Leeds, UI

\subsection{6/gutjnl-2018-BSGAbstracts.60}

Background Duodenal adenomas consist of sporadic and familial adenomatous polyposis (FAP) associated adenomas. Endoscopic mucosal resection (EMR) is the recognised technique when considering endoscopic removal of these lesions, but outcomes from large studies are lacking. Leeds Teaching Hospitals (LTHT) is a large tertiary centre that has a local catchment area of more than 800000 people, and to our knowledge this is the largest UK cohort assessing duodenal EMR outcomes.

Methods Retrospective data collection was performed of all patients who underwent duodenal EMR over a 17 year period at LTHT. We collected data on patient demographics, lesion characteristics and outcomes including significant complications, recurrence and surgery-free survival. Procedures were performed by a single advanced therapeutic endoscopist or an endoscopy fellow under supervision.

Results A total of 98 patients underwent EMR (sporadic $n=23$, FAP $\mathrm{n}=75$ ). Median adenoma size was $12.5 \mathrm{~mm}$ (IQR 9.0$30.0 \mathrm{~mm}$ ), with $46.9 \%$ removed en-bloc. Standard EMR was performed in 87 procedures, and 'pull-within snare' technique in the remaining 11 procedures. Final lesion histology was TA/TVA with LGD $(n=80)$, TA/TVA with HGD $(n=12)$, intra-mucosal cancer $(n=3)$ and in 3 cases data was missing. Patients with FAP were significantly younger with a median age of 49 years $(\mathrm{p}<0.001)$.

The overall complication rate was $12.4 \%$. One $(1.0 \%)$ patient had an intra-procedural bleed which could not be managed endoscopically, delayed bleeding occurred in 6 cases (6.2\%) and perforation occurred in 5 cases (5.2\%), 3 (3.1\%) of which could not be managed endoscopically. Following univariate analysis, 'pull-within snare' technique $(p=0.03)$, piecemeal resection $(p=0.002)$, and increasing polyp size $(p=0.003)$ were significantly associated with complications. Adenoma recurrence at first follow up was $25.0 \%$.

Surgery was required in 6 patients (6.2\%) within 24 months of their EMR, $4(4.1 \%)$ cases for adenomas $>30 \mathrm{~mm}, 2(2 \%)$ cases for 10-29 $\mathrm{mm}$ and no cases for adenomas $<10 \mathrm{~mm}$.

Conclusion This is the largest cohort in the UK pertaining to duodenal EMR outcomes, with success, recurrence and complications similar to other world leading endoscopy centres. Adverse outcomes are associated with increasing lesion size, piecemeal resection and EMR technique.

\section{PTH-040 ENDOSCOPIC DUODENAL MUCOSAL RESURFACING IN TYPE 2 DIABETES - A SINGLE CENTRE EXPERIENCE}

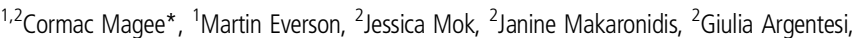
${ }^{1}$ Sally Thorpe, ${ }^{2}$ Pajany Vythelingum, ${ }^{3}$ Andrea Pucci, ${ }^{3,4}$ Rachel Batterham, ${ }^{1,5}$ Rehan Haidry. ${ }^{1}$ Department of Gastroenterology, University College London Hospitals, London, UK; ${ }^{2}$ Department of Metabolism and Experimental Therapeutics, University College London, London, UK; ${ }^{3}$ Bariatric Centre for Weight Management and Metabolic Surgery, University College London Hospitals, London, UK; ${ }^{4}$ Centre for Obesity Research, Department of Medicine, University College London, London, UK; ${ }^{5}$ Division of Surgery and Interventional Science, University College London, London, UK
}

Introduction Evidence from bariatric surgical procedures (e.g. Roux-en-Y gastric bypass) suggests that the duodenum plays a crucial role in glycaemic control in patients with Type 2 Diabetes (T2DM). Duodenal mucosal resurfacing (DMR), a novel endoscopic therapy that resurfaces the duodenal mucosa via hydrothermal ablation, exerts an insulin-sensitising effect likely through modification of nutrient-mucosa signalling. DMR involves placing a catheter in the proximal duodenum distal to the papilla under endoscopic and fluoroscopic guidance. The duodenal mucosa is injected with saline to 'lift' it and a balloon is inflated with water and heated to $90^{\circ} \mathrm{C}$ to circumferentially ablate the mucosa for $10 \mathrm{~cm}$. We describe the first series of patient cases from the UK treated with DMR at at University College London Hospitals (UCLH).

Methods Cases were derived from two clinical studies: REVITA 1 a single arm, open-label multi-centre in T2DM, and the open-label phase of REVITA-2 an internationl, multicentre, randomised, double-blinded, sham controlled study in T2DM. Eligible paitents were adults with T2DM and HbA1c of $7.5 \%-10.0 \%$ on $\geq 1$ oral glucose lowering medication. Baseline blood tests were collected and following a 4-6 week runin period, patient's were treated with the DMR procedure. Patients received dietetic advice were given a specific 2 week post procedure diet. Blood tests were analysed at regular intervals following the procedure to assess changes in HbA1c over time. Hepatic transaminases were also measured and calulations were made for Homeostasis Model Assessment index (HOMA-IR) - a measure of insulin resistance. Information on adverse events was recorded at each visit.

Results A total of 11 patients ( $\mathrm{n}=5$ female, $58 \pm 11$ years) were treated with DMR at UCLH from May 2015 - Dec 2017. Mean $( \pm$ SEM) change in HbA1c at 3 months post DMR was $0.5 \%( \pm 0.2)$ for all patients and $-0.7 \%$ $( \pm 0.2$ when one patient was excluded for whom gliclazide had to be stopped due to symptomatic hypoglycaemia in the month following DMR. A total of $5 / 11$ patients completed 6 and 12 months of follow-up with mean changes in HbA1c of $-1.3 \%$ and $-1.5 \%$ respectively. Favourable directional changes were also observed in HOMA-IR and hepatic transaminases. Patients tolerated the procedure well and no SAEs or UADEs were reported. Two of the eleven patients (18\%) patients experiencing transient constipation.

Conclusion Based on our centre's experience, DMR appears to provide a safe and effective method of improving glycaemic control in patients with T2DM. We contintue to enrol patients in the REVITA-2 trial and the results of this study will be crucial in determining the effectiveness and durability of DMR.

\section{PTH-041 PROSPECTIVE STUDY OF THE DIAGNOSTIC YIELD FROM 20G FNB NEEDLE IN ROUTINE UPPER GI AND HPB LESIONS}

Nazar Aslam*, Elaenor Liu, Sameer Shaktawat, Venkat Mahesh. Blackpool Victoria Hospital, Blackpool, UK

\subsection{6/gutjnl-2018-BSGAbstracts.62}

Introduction Improvements in the EUS FNA and FNB needle design and optimisation of tissue acquisition techniques have resulted in better diagnostic yield (DY). In addition, recently there has been considerable interest in histological processing of tissue acquired using newer 'core' FNA needles. At BSG 2016 we provided preliminary data on DY of tissue acquired using 
standard FNA needle. We hereby present data on yield of 20G Cook EchoTip Procore ${ }^{\mathrm{TM}}$ FNB needle.

Aim To compare the diagnostic yield of 20G FNB needle and compare the incremental yield of histological over cytological preparation of tissue acquired.

Methodology Prospective non-blinded randomised study. All patients undergoing EUS guided FNA/FNB from January 2016 to January 2018, for all upper GI and HPB lesions were included. FNB was performed using Cook Echotip Procore 20GTM needle, Olympus EU-ME2TM processor and linear echoendoscopes was used. All patients had 2 or 4 passes done with the same needle, specimens from each pass were randomly collected in BD Cytorich $^{\mathrm{TM}}$ or Formalin. Each preservative had either 1 or 2 passes of material. Only C5 malignancy diagnosis from the primary pathologist or from second expert opinion with MDT acceptance was considered positive for cancer, other non-malignant conditions was considered as positive for statistical purposes only when clear diagnosis was offered by pathologist.

Results In total, 147 patients had EUS guided sampling and 111 patients had EUS FNB with 20G FNB needle. $68 \mathrm{M}: 43$ F. Average age was 66.7 years $( \pm 11.3)$. Of these, 102 had samples both for cytology and histology. Final diagnosis was made in $92(90.2 \%)$ patients. Pancreatic cancer was seen in $52(51 \%)$ patients, cholangiocarcinoma in 7 (6.7\%), NET/ GIST in $11(10.8 \%)$, Lymphoma in 11 (10.8\%), malignant nodes in 6 (5.9\%), other cancers (e.g. rhabdomyosarcoma) in 6 (5.9\%), benign (e.g. chronic pancreatitis, autoimmune pancreatitis, paraganglioma, reactive nodes etc) in 8 (7.7\%), and no diagnosis was made in 10 (9.8\%).

Histological processing provided answers in 87 (78.3\%) patients with combined cytological and histological processing providing diagnosis in the rest of 15 (13.5\%) with overall diagnostic yield of $91.9 \%$ (102 patients).

Conclusion Our study results show that adequate samples can be obtained for histological processing with Cooke Procor$\mathrm{e}^{\mathrm{TM}}$ FNB needles with good cumulative diagnostic yield (92\%) and significant diagnostic yield from preferentially processing samples for histological assessment rather than standard cytology, possibly due to better preservation of tissue architecture and cell morphology. Limitations include non-specialist reporting cytopathologist and non-randomised retrospective design.

\section{PTH-042 COLORECTAL STENTING AS A BRIDGE TO SURGERY: A DECADE OF SINGLE CENTRE SUCCESS}

Lindsay Steward*, Miriam Manook, Waveney Stafford, Fiona Taylor, Stefano Andreani, Alan Watson. Queen's Hospital, Romford, Little Berkhamsted, UK

\subsection{6/gutjnl-2018-BSGAbstracts.63}

Introduction Colonic stenting in obstructing left sided colorectal cancer (CRC) using self-expanding metallic stents has been utilised as a conservative management intervention, as well as a bridge to definitive surgery. Despite high reported success rates technically and clinically of stent deployment, as well as improved rates of primary anastomosis, controversy remains about the use of this procedure as a bridge to surgery. We report on a decade of single centre experience.

Methods Retrospective analysis of patients requiring urgent colorectal cancer stenting as a bridge to surgery between 2006 and 2017. Primary outcomes measured were clinical and technical success. Secondary outcomes recorded were survival, complications, primary anastomosis and stoma formation

Results 36 patients underwent CRC stenting with the intention of being a bridge to elective surgery, of whom $44 \%$ were female. Mean age was 65.7 years. The majority of cases $(84 \%)$ were undertaken by one of the 3 main operators. At staging, 9 patients (25\%) were T3; 17 (47.3\%) T4. 25\% of patients were ASA 3. Technical success was reported in $89 \%$ of cases and clinical success $86 \%$. At elective surgery primary anastomosis was achieved in $61 \%$. 5 reported early complications, including 2 clinical perforations and 2 radiological perforations. Mean survival post stenting procedure was 24 months (range 3-55 months), with death due to metastatic CRC. There were no deaths within 30 days.

Conclusions We demonstrate excellent technical success with this treatment modality. By temporising patients using endoscopic stenting, patients are optimised for elective surgery by colorectal surgeons with good primary anastomosis rates, and excellent post procedure mortality.

\section{PTH-043 ADULT COELIAC DISEASE REMISSION ASSESSMENT: DOES A D1 BIOPSY INCREASE THE DETECTION OF VILLOUS ATROPHY?}

${ }^{1}$ Lauren Marks*, ${ }^{1}$ Michelle Lau, 'Matthew Kurien, ${ }^{2}$ Marios Hadjivassiliou, 'Peter Mooney, ${ }^{3}$ ProfSimon Cross, ${ }^{1}$ David Sanders. ${ }^{1}$ Academic Unit of Gastroenterology, Royal Hallamshire Hospital, Sheffield, UK; ${ }^{2}$ Department of Neurology, Royal Hallamshire Hospital, Sheffield, UK; ${ }^{3}$ Department of Pathology, Royal Hallamshire Hospital, Sheffield, UK

\subsection{6/gutjnl-2018-BSGAbstracts.64}

Introduction The duodenal bulb (D1) has been shown to be a sensitive site for detecting villous atrophy (VA) in newly diagnosed coeliac disease (CD), however there is a scarcity of data from those with established CD. In patients with established $\mathrm{CD}$, we aim to establish whether D1 biopsies improved the identification of VA compared to biopsies from the second part of the duodenum (D2) alone.

Methods 251 patients with established CD were prospectively recruited from the endoscopy department at the Royal Hallamshire Hospital between 2013 and 2017. These patients were undergoing repeat gastroscopy to assess dietary adherence. All patients underwent a gastroscopy, with one biopsy taken from the duodenal bulb and four from D2. Biopsies were classified according to Marsh criteria. We assessed concordance of histology between the D1 and D2 sites, and 95\% confidence intervals were calculated for all results using a binominal distribution.

Results 251 patients were recruited $(70.5 \%$ female, age range 17-81 years, median age 53 years) having been on a glutenfree diet for a median duration of 6 years. Concordant results: $35.1 \%$ ( $n=88,95 \% \mathrm{CI}: 29.16$ to 40.96$)$ had normal duodenal biopsies in both D1 and D2; 32.3\% ( $\mathrm{n}=81,95 \%$ CI: 26.49 to 38.05$)$ had VA in D1 and D2; $18.3 \%(n=46$, 95\% CI: 13.54 to 23.11 ) had raised intra-epithelial lymphocytes (IELs) only in both D1 and D2. Disconcordant results: $4.4 \%(\mathrm{n}=11,95 \% \mathrm{CI}: 1.85$ to 6.91$)$ had VA in D1 but not D2; $2.4 \%(n=6,95 \% \mathrm{CI}: 0.50$ to 4.28$)$ had raised IELs in D1 but normal histology in D2. $2.8 \%(n=7,95 \%$ CI: 0.75 to 4.83) had VA in D2 but normal histology in D1; $4.8 \%$ $(\mathrm{n}=12,95 \% \mathrm{CI}: 2.14$ to 7.42$)$ had IELs in D2 but normal histology in D1.

Conclusions VA was confined to the duodenal bulb in $4.4 \%$ of patients with established CD. Thus a D1 biopsy in addition 